\title{
Vigilancia activa en carcinoma papilar tiroideo de bajo riesgo (parte II): historia de progresión de la enfermedad, calidad de vida y costo-efectividad
}

\author{
Active surveillance of low-risk papillary thyroid carcinoma (part II): \\ history of disease progression, quality of life and cost effectiveness
}

\author{
Mario Tapia C. ${ }^{1,4}$, Felipe Cardemil M..$^{2,3}$
}

\section{Resumen}

El aumento dramático en la incidencia del cáncer de tiroides de las últimas décadas, el cual se debe principalmente a la mayor detección de microcarcinomas papilares de tiroides (MCPT), ha generado un aumento considerable de los costos asociados a la atención del cáncer de tiroides. A pesar de este aumento de la incidencia a nivel mundial, la mortalidad asociada a estos tumores se ha mantenido sin cambios significativos. En consecuencia, varios grupos de investigadores han sugerido que se ha producido un sobrediagnóstico y un sobretratamiento de los carcinomas papilares de tiroides (CPT) de bajo riesgo. La vigilancia activa (VA) ha surgido como una nueva alternativa terapéutica para estos tumores, con excelentes resultados oncológicos, menores tasas de complicaciones y que podría ayudar a reducir los costos económicos. No obstante, a pesar de los alentadores resultados de la VA, su implementación en la práctica depende de muchos aspectos entre los cuales se encuentran las características radiológicas del tumor, las características clínicas del paciente, factores psicosociales y socioeconómicos. En esta revisión nos enfocaremos en describir la evidencia acumulada hasta la fecha respecto de la historia natural de la cinética del CPT, costo-efectividad de VA, y las limitaciones actuales para su implementación.

Palabras clave: Vigilancia activa, carcinoma papilar tiroideo, bajo riesgo, microcarcinoma papilar tiroideo, cáncer de tiroides.

\begin{abstract}
The dramatic increase in the incidence of thyroid cancer in recent decades, which is primarily due to increased detection of papillary thyroid microcarcinomas (PTCM), has led to a significant increase in the costs associated with thyroid cancer care. Despite this increase in the worldwide incidence, the mortality associated with these tumors has remained unchanged. Consequently, several groups of researchers have suggested that low-risk papillary thyroid carcinomas (PTC) have been overdiagnosed and overtreated. Active surveillance (AS) has emerged as a new therapeutic alternative for these tumors, with excellent oncological results, lower complication rates, and which could help reduce economic costs. However, despite the encouraging results of AS, its implementation in practice depends on many aspects including the radiological characteristics of the tumor, the clinical characteristics of the patient, psychosocial factors and socioeconomic factors. In this review we will focus on describing the evidence accumulated to date regarding the natural history of PTC kinetics, cost effectiveness of VA, and current limitations to its implementation.
\end{abstract}

Keywords: Active surveillance, papillary thyroid carcinoma, low risk, papillary thyroid microcarcinoma, thyroid cancer.
'Servicio de

Otorrinolaringología,

Complejo Asistencial Dr.

Víctor Ríos Ruiz. Los Ángeles,

Chile.

${ }^{2}$ Departamento de Oncología

Básico-Clínica y Departamento de Otorrinolaringología,

Facultad de Medicina,

Universidad de Chile.

Santiago, Chile

${ }^{3}$ Departamento de

Otorrinolaringología, Clínica

Las Condes. Santiago, Chile.

${ }^{4} \mathrm{Head}$ and Neck Surgical

Oncology Department, Peter

MacCallum Cancer Centre.

Melbourne, Australia.

Los autores declaran no tener conflictos de interés.

Recibido el 6 de agosto de 2020. Aceptado el 7 de octubre de 2020.

Correspondencia:

Mario Tapia C.

Servicio de

Otorrinolaringología

Complejo Asistencial

Dr. Víctor Ríos Ruiz.

Los Ángeles, Chile.

Email: mtapiac21@gmail.com 


\section{Introducción}

El carcinoma papilar de tiroides (CPT) es el tipo más común de cáncer de tiroides y su incidencia ha incrementado dramáticamente en las últimas décadas ${ }^{1}$. Estos incrementos se deben principalmente al aumento en la detección de carcinomas papilares menores a $2 \mathrm{~cm}$ de tamaño, mientras que la incidencia de los carcinomas papilares mayores a $2 \mathrm{~cm}$ y otros tipos de cáncer de tiroides se han mantenido estables ${ }^{1}$. Los carcinomas papilares con tamaño menor o igual a $1 \mathrm{~cm}$ denominados microcarcinomas papilares (MCPT) representan el 39\% de los casos de cáncer de tiroides en Estados Unidos $^{1}$ y el 43,1\% de los casos en Corea del $\mathrm{Sur}^{2,3}$. A pesar de este aumento de la incidencia a nivel mundial, la mortalidad asociada a estos tumores se ha mantenido sin cambios significativos $^{4,5}$. Por otro lado, estudios basados en autopsias han demostrado la presencia de CPT ocultos en hasta un tercio de los pacientes fallecidos por enfermedades no relacionadas con patologías tiroideas ${ }^{6}$.

En vista de estos datos, varios grupos de investigadores han sugerido que se ha producido un sobrediagnóstico y un sobretratamiento de carcinomas papilares de bajo riesgo ${ }^{1-3}$. Actualmente la gran mayoría de los pacientes con un cáncer de tiroides recién diagnosticado son tratados quirúrgicamente, y el número de pacientes con parálisis de las cuerdas vocales e hipoparatiroidismo posoperatorio ha aumentado $^{2}$. Aunque el tratamiento quirúrgico ha sido el estándar durante años, la vigilancia activa (VA) ha surgido como una alternativa terapéutica que implica la observación periódica y regular con el objetivo de identificar una minoría de pacientes que progresarán clínicamente y que probablemente se beneficiarán de la cirugía de rescate ${ }^{7}$. Además, el retraso en el tratamiento quirúrgico de pacientes adecuadamente seleccionados con carcinomas papilares de tiroides (CPT) de bajo riesgo no se ha asociado con mayor riesgo de enfermedad persistente o recurrente en comparación con la intervención inmediata ${ }^{8,9}$. Los datos de los primeros investigadores que llevaron a cabo ensayos de VA en los CPT de Japón, y más tarde de diversos países del mundo, han demostrado que la VA es una alternativa segura para el manejo de los CPT papilares de bajo riesgo ${ }^{8,9}$.
Los costos médicos son otro aspecto importante a considerar respecto al tratamiento de los CPT. Lubitz y cols. advirtieron que el costo social de la atención del cáncer de tiroides en los EE. UU. fue de 1.600 millones de dólares en 2013 y podría aumentar a 3.500 millones de dólares en 2030 si continúa la tendencia al alza de su tasa de incidencia ${ }^{10}$. Los costos médicos son un tema muy importante para cualquier sociedad y también constituye un factor de preocupación para los pacientes ${ }^{10}$.

Durante las últimas décadas no existía suficiente conocimiento del comportamiento biológico de los CPT, y se ha reportado que muchos de estos CPT pequeños tienen características patológicas similares a los de mayor tamaño tales como, la presencia de metástasis en los ganglios linfáticos cervicales (LN), extensión extratiroidea (ETE) y multifocalidad $^{11,12}$. Estos hallazgos han llevado a un mayor número de diagnósticos y cirugías para ellos, sin embargo, en gran medida gracias a los extensos estudios de VA con largos periodos de seguimiento, actualmente sabemos que la gran mayoría de los MCTP y CPT de bajo riesgo tienen una naturaleza indolente $e^{2,13,14}$.

A pesar de los alentadores resultados de la VA su implementación en la práctica clínica está fuertemente influenciada por factores psicosociales, particularmente en los países menos desarrollados económicamente en los que según reportes recientes sólo alrededor de una cuarta parte de los pacientes han estado dispuestos a aceptar esta modalidad ${ }^{15}$. En esta revisión nos enfocaremos en describir la evidencia acumulada hasta la fecha respecto a la historia natural del CPT, los aspectos socioeconómicos relacionados a sus alternativas terapéuticas actualmente aceptadas, y analizar las limitaciones actuales para la implementación de la VA.

\section{Historia natural del carcinoma papilar de tiroides}

Se ha identificado que los MCPT pueden presentar un aumento de volumen tumoral (VT) en una etapa temprana mientras el diámetro máximo permanece sin cambios significativos ${ }^{16}$. En consecuencia, se ha añadido el aumento del VT en al menos un 50\% como definición del crecimiento del tumor en algu- 
nos estudios, en los cuales se ha identificado que el MCPT que inicialmente es verticalmente delgado (más alto que ancho) se vuelve globular con el tiempo, generando que el VT cambie sin alterar el diámetro máximo ${ }^{16}$. Este hallazgo sugiere que la medición del VT resultaría más sensible que la medición del diámetro máximo para identificar el crecimiento tumoral, y pudiese ser mejor para identificar una progresion temprana en MCPT.

Un estudio realizado en Memorial Sloan Kettering Cancer Center (MSKCC) presentó resultados similares con un aumento del VT lineal hasta 5 años ${ }^{17}$, hallazgo que también fue identificado en un estudio multicéntrico coreano $^{18}$. Un estudio reciente realizado en Corea del Sur que incluyó a 273 pacientes con CPT reportó que un crecimiento tumoral rápido, con tiempo de duplicación de VT menor a 5 años, se asoció significativamente a pacientes menores de 50 años y a la presencia de macrocalcificación ${ }^{19}$. En síntesis, los autores concluyen que la velocidad de crecimiento del MCPT evaluada por la medición del VT podría predecir tempranamente qué pacientes finalmente progresarán a largo plazo. Sin embargo, hasta ahora la cinética tumoral solo ha sido estudiada durante un período de tiempo limitado. Aunque el MCPT muestre un crecimiento lineal de hasta 5 años, no está claro si tal velocidad de crecimiento se mantendrá constante más allá de este periodo, y en última instancia desarrolle una enfermedad clínicamente significativa que requiera cirugía.

Por otro lado, Miyauchi y cols. realizaron el cálculo de la tasa de progresión de la enfermedad (crecimiento tumoral y/o desarrollo de adenopatías) durante 10 años bajo VA para cada grupo de edad al momento del diagnóstico de su CPT, entre los 20 a 70 años. Las estimaciones de progresión de enfermedad fueron de $48,6 \%, 25,3 \%, 20,9 \%, 10,3 \%, 8,2 \%$, y $3,5 \%$ para pacientes de $20,30,40,50,60$ y 70 años, respectivamente. La probabilidad de progresión de enfermedad en pacientes de 20 años y los 30 pudiesen ser demasiado altos para que la vigilancia activa sea aceptada como alternativa terapéutica, sin embargo, desde otra perspectiva estos valores indican que más de la mitad de los pacientes de 20 años y alrededor de $75 \%$ de los pacientes de 30 años no requerirían cirugía en su vida ${ }^{20}$.
Para evaluar la cinética del volumen del tumor, Miyauchi y cols. ${ }^{21,22}$ propusieron la tasa de duplicación, que es distinto al tiempo de duplicación utilizada en los otros estudios mencionados. Los autores establecen que a pesar de que el tiempo de duplicación es una herramienta bien validada para evaluar la cinética del volumen del tumor, tiene dos inconvenientes. En primer lugar, si los tumores se reducen con el tiempo, los tiempos de duplicación de estos tumores se darían en valores negativos (menores a 0 ) creando una discontinuidad entre los valores positivos y negativos. Además, el tiempo de duplicación disminuye cuando la actividad de crecimiento del tumor aumenta, lo que indica que la magnitud del tiempo de duplicación es opuesta a la de la tasa de crecimiento del tumor. La tasa de duplicación puede utilizarse para resolver estos problemas y reflejar la actividad de crecimiento del tumor porque indica el número de duplicaciones que se producen por unidad de tiempo. Los autores calcularon las tasas de duplicación del volumen del tumor por año en 169 MCPT durante VA y las clasificaron en cuatro categorías: mayor a 0,5 (crecimiento rápido), 0,1 a 0,5 (crecimiento lento), $-0,1$ a 0,1 (crecimiento estable) y menor a $-0,1$ (disminución del crecimiento). De los $169 \mathrm{MCPT}$ estudiados, $3 \%, 22,5 \%, 57,4 \%, 17,1 \%$ presentó un crecimiento rápido, un crecimiento lento, un crecimiento estable y una disminución del crecimiento, respectivamente ${ }^{22}$. Además, el $17 \%$ de los MCPT disminuyó su VT durante la vigilancia activa. La incidencia de MCPT con crecimiento rápido y lento estaba inversamente relacionada con la edad de los pacientes; $40 \%$ en los pacientes menores de 40 años, $29 \%$ en los de 40 a 59 años, y 17\% en los de 60 años o más.

En el mismo estudio los autores también calcularon la "hipotética" tasa de duplicación del VT de los MCPT antes de la presentación, suponiendo que una sola célula cancerosa de 10 micrómetros $(\mu \mathrm{m})$ estuviese presente al nacer y crezca a un ritmo constante. Este valor indica la menor tasa de crecimiento necesaria para que una célula neoplásica crezca hasta el tamaño del tumor en la presentación. Curiosamente, las tasas de duplicación hipotéticas eran mucho más altas que las tasas de duplicación observadas durante la VA después de la pre- 
sentación $^{22}$. Por lo tanto, concluyeron que el crecimiento de las células cancerosas se produce después del nacimiento y no en el momento del nacimiento, que la tasa de duplicación real del tumor debería ser incluso mayor que la tasa de duplicación hipotética, y sus resultados sugieren que un MCPT crecería más rápidamente antes de la presentación clínica, y que después de la presentación, la actividad de crecimiento disminuiría significativamente o se convierte en cero, o incluso menor que cero (contracción del tumor), lo que podría corresponder a un curso natural de MCPT.

\section{Evaluación económica}

Dado que los costos médicos y la práctica clínica son diferentes en cada país, es necesario realizar evaluaciones económicas específicas para cada uno. En un estudio en el que se analizó la costo-efectividad de VA para MCPT en Hong Kong se reportó un ahorro de costos durante los primeros 16 años de VA en comparación con la cirugía temprana independientemente de la edad del paciente, después de la cual la VA seguía siendo costo-efectiva a pesar de significar un costo más elevado después de 16 años $^{23}$. Los autores concluyeron que la calidad de vida de los pacientes sometidos a VA puede ser lo suficientemente superior como para compensar en caso de presentarse una reducción de su costo-efectividad. En otro estudio realizado en Japón se calcularon los costos médicos de la cirugía inmediata y la VA bajo el Sistema de Seguro de Salud Japonés, reportándose que el costo a 10 años de la cirugía inmediata era 4,1 veces mayor que el de la VA (928.094 frente a 225.695 yenes/paciente $)^{24}$. El costo de la cirugía de conversión en caso de progresión de enfermedad durante VA fue incluido en este grupo, y los costos de la cirugía para la recidiva y el tratamiento posoperatorio se incluyeron en el grupo de cirugía inmediata. En Estados Unidos, Venkatesh y cols. ${ }^{25}$ también calcularon la costo-efectividad de la VA utilizando un caso base de un paciente de 40 años con CPT de bajo riesgo tratable mediante lobectomía. Demostraron que la costo-efectividad dependía en gran medida de la disminución de la calidad de vida de cada paciente que se producía al diagnosticar el MCPT y de la expectativa de vida remanente del paciente. Ellos concluyeron que la lobectomía temprana es más costo-efectiva que la VA en los pacientes con MCPT en los que el diagnóstico se asocia con una disminución de la calidad de vida.

Por lo anteriormente expuesto, debido a que la probabilidad de progresión de la enfermedad a lo largo de la vida durante la VA está relacionada con la década de la vida en la cual se diagnostique, la edad es un factor importante para determinar la costo-efectividad de la VA en CPT de bajo riesgo ${ }^{20}$. Dado que la tasa estimada de progresión de la enfermedad a lo largo de la vida es del $60 \%$ para los pacientes de 20 años, la intervención quirúrgica temprana resultaría más costo-efectiva en estos pacientes.

\section{Calidad de vida}

A pesar del relativo éxito del tratamiento del cáncer de tiroides comparado con otros tumores malignos, los sobrevivientes de cáncer de tiroides tratados de manera quirúrgica reportan disminuciones significativas en la calidad de vida durante los primeros 5 años después del diagnóstico, incluso peor que pacientes con otros diagnósticos menos favorables como el cáncer de mama o de colon ${ }^{26}$. Se desconoce qué parte de esta disminución es atribuible al tratamiento del cáncer de tiroides que incluye cirugía y frecuentemente la necesidad de reemplazo hormonal a largo plazo, y si los pacientes tratados con VA experimentan disminuciones similares.

$\mathrm{La}$ ansiedad de los pacientes sobre la progresión de la enfermedad durante la VA de MCPT ha sido reportada como la razón más común para la realización de cirugía de conversión ${ }^{7,9,16,27}$. Los pacientes bajo VA reportan disminución del deseo sexual en comparación con los que se someten a la cirugía, lo que puede estar relacionado con la ansiedad respecto al diagnóstico de cáncer ${ }^{28}$, sin embargo, la ansiedad del paciente durante VA puede disminuir con el tiempo, por lo que la ansiedad inicial a la estrategia no constituye una contraindicación absoluta $^{29}$.

Un estudio conjunto entre el Instituto Dartmouth en Estados Unidos y el Hospital Kuma en Japón, evaluó específicamente la experiencia personal de 249 pacientes some- 
tidos a VA ${ }^{29}$. Mediante una combinación de encuestas y entrevistas a pacientes, revelaron que los pacientes en general se preocupan por la recurrencia del cáncer, las metástasis y la necesidad de una intervención quirúrgica en un grado similar al de los pacientes que se someten a una cirugía. Es importante destacar que el $80 \%$ de los pacientes estuvieron de acuerdo o muy de acuerdo en que su decisión de someterse a VA se ajustaba a sus valores personales. Además, el 83\% estuvo de acuerdo o muy de acuerdo en que la elección de la VA fue la mejor decisión para ellos ${ }^{29}$. Concluyeron que estos resultados favorables se debían no sólo al éxito del tratamiento, sino también a la confianza de los médicos en este abordaje terapéutico. Además, una gran proporción de pacientes (77\%) no conocían la VA como modalidad de tratamiento antes de que se les ofreciera. Este estudio destacó que la experiencia del paciente con la VA no tiene un impacto perjudicial en el bienestar psicológico y es comparable a la de los pacientes que se someten a una intervención quirúrgica.

La preocupación que un paciente puede experimentar debido a la convivencia con un cáncer activo si bien es comprensible y razonable, no significa una contraindicación de la VA como una alternativa terapéutica. Los autores concluyeron que frecuentemente la explicación respecto a que la cirugía puede realizarse en un futuro con igual efectividad a si hubiese sido realizada inicialmente es útil para que los pacientes consideren sus alternativas adecuadamente ${ }^{29}$.

Además, la ansiedad relacionada a la VA no sólo ocurre entre los pacientes sino también entre los médicos. Dos estudios australianos demostraron que muchos médicos dudan en aceptar y ofrecer la $\mathrm{VA}^{30,31}$, sin embargo, la mayoría de los médicos incluidos en los estudios no tenían experiencia con este manejo. En contraste, en el Hospital Kuma, la incidencia de pacientes que eligieron la VA fue inicialmente de 30\% entre 1993 y 1997, sin embargo, la incidencia aumentó con el tiempo alcanzando el $88 \%$ en 2014 y más de un $95 \%$ en el año $2018^{32}$. Las razones de estos resultados son que la efectividad y la seguridad de la VA se han establecido de forma más significativa que antes, y las incidencias de eventos desfavorables como la parálisis de las cuerdas vocales y el hipoparatiroidismo fueron significativamente mayores en el grupo de cirugía inmediata que en el grupo de vigilancia activa, a pesar de que todas las cirugías fueron realizadas por cirujanos endocrinos experimentados en el Hospital Kuma, un centro de derivación para patologías tiroideas $^{33}$. Estos dos factores influyeron fuertemente en la actitud de los médicos que trataban a los pacientes con MCPT para recomendar la vigilancia activa de estos pacientes.

En el Hospital de Kuma en Japón, se entrega un folleto que explica la seguridad y los resultados favorables de la VA a los pacientes que tienen nódulos sospechosos de CPT en la ecografía antes del examen citológico, y un segundo folleto se entrega a los pacientes que han estado bajo VA durante mucho tiempo con objeto de mantener niveles de ansiedad bajos. El equipo de MSKCC y el equipo coreano elaboraron un programa educacional colaborativo denominado "Elección del tratamiento para el cáncer de tiroides" para apoyar la comunicación entre médicos y pacientes ${ }^{34}$, y reportaron que los pacientes que recibieron $\mathrm{di}$ cha ayuda tenían más probabilidades de elegir la VA que los que no la recibieron ${ }^{34}$.

Como se señaló anteriormente, la calidad de vida relacionada con el temor a la progresión de la enfermedad no parece ser diferente entre los pacientes quirúrgicos y los que se encuentran bajo VA. En un estudio reciente se comparó la calidad de vida de los pacientes con MCPT bajo VA con la de los que se sometieron a una lobectomía utilizando tres cuestionarios diferentes, en el cual el análisis de la ansiedad y el miedo relacionados con la progresión de la enfermedad no mostró diferencias significativas entre los dos grupos. Además, después de ajustar la edad, el sexo y los niveles de TSH en suero, los pacientes que se sometieron a la lobectomía mostraron más problemas de salud que pueden estar relacionados con la cirugía, que los que fueron tratados mediante $\mathrm{VA}^{28}$. Un estudio realizado en Japón también reportó que el nivel de preocupación por el cáncer en los pacientes con VA eran comparables a las de los pacientes tratados activamente ${ }^{29}$.

Por otro lado, las complicaciones quirúrgicas tienen un gran efecto en la calidad de vida. Se han reportado tasas de incidencia acumulada de complicaciones quirúrgicas más elevadas en pacientes que fueron sometidos a cirugía 
temprana que los que fueron tratados mediante VA inicialmente ${ }^{35}$. Sin embargo, cuando los pacientes que recibieron cirugía tardía fueron considerados como un subgrupo, se reportó que la tasa de complicaciones era más alta entre ellos, que entre los que se sometieron a una cirugía temprana $^{35}$. La mayoría de los MCPT tienen una probabilidad muy leve de extensión extratiroidea macroscópica y/o metástasis cervical ganglionar clínica, y pueden ser tratados con lobectomía. No obstante, si se desarrolla metástasis linfática durante la VA los pacientes deben recibir un tratamiento quirúrgico más extenso $^{36}$ que puede resultar en un aumento del riesgo de complicaciones. Por lo tanto, pudiese ser más probable que la cirugía tardía durante la VA de la MCPT resulte en una cirugía más extensa que en la cirugía inmediata y, en consecuencia, resulte en una disminución de la calidad de vida relacionada con el aumento de las complicaciones quirúrgicas ${ }^{36}$.

Dada la indolencia de los MCPT en los pacientes que son candidatos a VA, la elección entre realizar una cirugía temprana o vigilancia activa es en gran medida una decisión sobre la calidad de vida que debe ser compartida entre el paciente y el médico. A fin de orientar al paciente en esta discusión, el médico tratante debe tener en cuenta la experiencia del paciente y debe ser consciente de los sesgos y supuestos intrínsecos que pueden llevar al médico a favorecer fuertemente una alternativa sobre otra. A pesar de que se evita el riesgo quirúrgico y la falta de un beneficio significativo de supervivencia, el impacto psicológico potencial de vivir con cáncer en el paciente puede impedir que el médico perciba la VA como una alternativa viable. Las investigaciones en curso tratan de definir mejor la experiencia del paciente durante el proceso de VA e identificar los obstáculos para la aplicación de esta modalidad de tratamiento.

\section{Eventos desfavorables de cirugía temprana y vigilancia activa}

Griffin y cols. realizaron una revisión retrospectiva de 681 pacientes con cáncer de tiroides tratados quirúrgicamente entre el año 2003 y 2012, con objeto de determinar la proporción de pacientes con CPT que habrían cumplido criterios para ser sometidos a vigilancia activa, y también determinar el número total de cirugías y complicaciones que podrían haber sido evitadas. En este estudio se identificó un total de 56 pacientes que cumplían criterios para VA y que fueron tratados quirúrgicamente, reportándose 6 casos de hipoparatiroidismo temporal y 1 permanente, 1 caso de parálisis de cuerda vocal (PCV) temporal y 1 caso de PCV permanente ${ }^{37}$. Si bien las tasas de complicaciones notificadas fueron bajas, en el contexto de pacientes que podrían haber sido tratados mediante VA según su clasificación de riesgo, estas tasas de complicaciones podrían considerarse inaceptablemente altas debido a que podrían haber sido evitadas en su totalidad. Entre los 56 candidatos potenciales a VA, 5 pacientes $(8,9 \%)$ necesitaron una cirugía de revisión por recidiva local después de la cirugía inicial para el CPT de bajo riesgo ${ }^{37}$. Por lo tanto, se podrían haber evitado procedimientos quirúrgicos múltiples en estos pacientes de haber sido tratados inicialmente mediante $\mathrm{VA}^{37}$.

De manera similar, Oda y cols. compararon las incidencias de eventos desfavorables entre 2.153 pacientes con MCPT, entre los cuales 974 fueron tratados mediante cirugía inmediata y 1.179 se sometieron a $\mathrm{VA}^{35}$. El grupo de cirugía inmediata tuvo incidencias significativamente mayores de parálisis transitoria de las cuerdas vocales $(4,1 \%$ vs. $0,6 \%, p<0,0001)$, hipoparatiroidismo transitorio $(16,7 \%$ vs. $2,8 \%$, $\mathrm{p}<0,0001$ ), e hipoparatiroidismo permanente ( $1,6 \%$ vs. $0,08 \%, \mathrm{p}<0,0001)$. Dos pacientes $(0,2 \%)$ en el grupo de cirugía inmediata desarrollaron parálisis permanente de las cuerdas vocales, mientras que ninguno en el grupo de VA presentó esta condición. Además, 5 $(0,051 \%)$ de los pacientes del grupo quirúrgico requirieron un segundo procedimiento quirúrgico debido al desarrollo de recurrencia loco-regional. En contraste, 94 pacientes de 1.179 del grupo de VA fueron sometidos a cirugía por diversas razones después de iniciar la VA y sólo 1 paciente $(0,08 \%)$ requirió un segundo procedimiento quirúrgico debido a recurrencia local. Los autores concluyeron que el riesgo de desarrollar metástasis ganglionares en los pacientes sometidos a VA es similar al de los tratados con cirugía inmediata y con este último abordaje es posible evitar cirugías múltiples ${ }^{35}$. 
Jeon y cols. reportaron una serie de 8.808 pacientes con MCPT entre los cuales 12 de ellos $(0,14 \%)$ presentaban metástasis a distancia y 4 fallecieron debido a cáncer de tiroides ${ }^{38}$. Sin embargo, todos los pacientes que desarrollaron metástasis distantes tenían metástasis ganglionares cervicales clínicas (que en su mayoría involucraban el área cervical lateral) y un paciente tenía metástasis distantes clínicamente evidentes antes de la cirugía inicial. Por lo tanto, los que desarrollaron metástasis a distancia de la MCPT primaria ya tenían una enfermedad clínica avanzada y no estaban calificados como CPT de bajo riesgo. Además, en estos pacientes los retrasos en la cirugía mayor a 18 meses no se asoció con un mayor riesgo de recurrencia en comparación con los que se sometieron a una cirugía inmediata ${ }^{39}$. De la misma manera los pacientes tratados quirúrgicamente mediante hemitiroidectomía no presentaron mayor tasa de recurrencia $(3,8 \%)$ que los tratados mediante tiroidectomía total $(1,6 \%)$ durante los 8,5 años de seguimiento ${ }^{40}$, y el desarrollo de recidiva ganglionar cervical lateral fue poco frecuente en ambos grupos $(0,6 \%$ en el grupo tratado mediante hemitiroidectomía y 1,5\% en el grupo de tiroidectomía total). Además, los pacientes con MCPT que se habían sometido a una tiroidectomía total presentaron excelentes resultados clínicos independientes de asociarse a $\mathrm{RAI}^{41} \mathrm{o}$ de la terapia de supresión de tirotropina (TSH) posoperatoria $^{42}$.

\section{Biomarcadores}

Actualmente no hay datos que indiquen que algún hallazgo molecular impacte en la idoneidad de CPT de bajo riesgo para ser tratado mediante VA. Las mutaciones promotoras de BRAF y/o TERT son marcadores moleculares pronósticos conocidos en cáncer de tiroides, pero su rol en la progresión de la MCPT no ha sido establecido ${ }^{43,44}$. Aunque las mutaciones BRAF y TERT en general predicen fuertemente un mal pronóstico de los CPT de mayor tamaño, ninguno de los MCPT incluyendo los que mostraron crecimiento durante la VA han evidenciado tales mutaciones ${ }^{44}$.

Hirokawa y cols. ${ }^{45}$ reportaron que el índice Ki-67 en los MCPT que mostraban crecimien- to fue significativamente mayor que el de los MCPT que no crecieron. Este resultado es esperable ya que el índice puede evaluarse en muestras patológicas, pero no en las citológicas, por lo tanto, no puede utilizarse como biomarcador de la progresión durante la VA. Hasta la fecha no se han identificado marcadores útiles para predecir la progresión de la MCPT utilizando muestras citológicas.

La relación entre la supresión de la TSH y la progresión de los MCPT es controvertida. En un estudio se reportó que la TSH no era un marcador pronóstico de la progresión de los $\mathrm{MCPT}^{46}$, mientras que Kim y cols. ${ }^{47}$ reportaron que una elevación sostenida de la TSH plasmática sí se relaciona con la progresión del MCPT. En el primer estudio, los pacientes eran mayores y los investigadores utilizaron el aumento del diámetro máximo como criterio de progresión ${ }^{46}$. En el segundo, los pacientes eran más jóvenes y los autores utilizaron el aumento del VT mayor a 50\% como criterio de crecimiento tumoral ${ }^{47}$. Dado que los MCPT en el grupo de mayor edad rara vez progresa y el aumento del diámetro máximo aparentemente no es la medida más sensible, estas diferencias en la población y el diseño del estudio podrían explicar los diferentes resultados. Además, Ito y cols. ${ }^{9}$ reportaron que los niveles de TSH no fueron suprimidos a niveles normales bajos por administración de levotiroxina entre los pacientes más jóvenes, a pesar de que los MCPT en pacientes más jóvenes eran más propensos a crecer que en pacientes de mediana edad y mayores. Por lo tanto, es necesario realizar estudios con mayor número de pacientes y metodologías comparables para obtener conclusiones más definitivas.

Las extensas experiencias japonesas, coreanas y norteamericanas más recientes no han utilizado ningún marcador molecular como criterio de inclusión o exclusión, y se sospecha que probablemente una parte sustancial de los CPT sometidos a VA en cada una de estas series habrían albergado mutaciones BRAF. Un reciente análisis mutacional reveló que no hay diferencias mutacionales claras entre los microcarcinomas papilares indolentes y los que desarrollan metástasis ganglionares ${ }^{47}$, sin embargo, es posible que en el futuro se identifiquen mejores discriminadores moleculares del comportamiento clínico. 


\section{Aceptación de vigilancia activa}

La detección precoz de ciertos cánceres ha sido un objetivo importante de la atención oncológica moderna. Esta mentalidad se ha difundido entre el público de tal manera que la preferencia por el diagnóstico y el tratamiento temprano es difícil de superar. Esta constituye una barrera importante que debe ser superada cuando se aboga por una estrategia como la VA. El cáncer de próstata es una malignidad análoga que ha tenido que superar el doble reto del sobrediagnóstico y el sobretratamiento ${ }^{48} \mathrm{y}$ la detección temprana en los cánceres de próstata de bajo riesgo se convirtió en un problema después del uso generalizado del antígeno prostático específico ${ }^{48}$.

Las guías sobre el cáncer de próstata mencionan ahora explícitamente los efectos perjudiciales de la detección de esta malignidad en la población general y destacan la importancia de la sensibilización de la salud pública sobre los posibles daños de la identificación de los cánceres indolentes de etapa temprana ${ }^{49}$. Se debe hacer hincapié en la importancia de la adopción de decisiones compartidas para los pacientes a los que se les diagnostica un cáncer indolente, ya que la cirugía puede generar un daño innecesario. El cáncer de próstata ha demostrado que la VA es una estrategia esencial para una enfermedad con un gran reservorio subclínico de enfermedad, una larga historia natural, y excelentes tasas de supervivencia ${ }^{48}$. De esta manera es un modelo de cómo manejar las expectativas de los pacientes y del público en relación con el diagnóstico de un tumor indolente. En muchos sentidos, la VA del cáncer de próstata fue adoptada mas facilmente debido a que los riesgos de la cirugía superaban los riesgos del cáncer, sin embargo, lo contrario puede influir en el cáncer de tiroides; los pacientes son más jóvenes, la tiroidectomía tiene efectos adversos menos notorios y, por lo tanto, la aceptación de la VA es comprensiblemente más compleja. No todos los pacientes se sienten cómodos con el tratamiento de observación cuando se les presenta un diagnóstico de cáncer y, en consecuencia, muchos rechazarán la VA como una opción de tratamiento aceptable.

No obstante, debido a los nuevos datos respecto a los resultados de la VA tales como, la seguridad, las bajas tasas de recurrencia, el éxito de la cirugía tardía y, por último, la falta de mortalidad asociada a la vigilancia, la guía ATA 2015 incluye a la VA como una alternativa para el manejo de $\mathrm{CPT}^{50}$. Es importante señalar que la cirugía puede ofrecerse en cualquier momento del período de vigilancia y que las guías no limitan esta alternativa solo para pacientes con tumores menores de $1 \mathrm{~cm}$, sino a una lesión de bajo riesgo.

Según los estudios existentes, menos de la mitad de los pacientes elegibles para la VA elegirán esta estrategia en lugar de una cirugía inmediata $^{51}$. Los pacientes declaran que el potencial de diseminación metastásica del cáncer constituye una preocupación clave cuando se enfrentan a un diagnóstico de CPT de bajo riesgo $y$, aunque la aversión a la terapia de reemplazo hormonal de por vida es un factor importante, generalmente no preferirán la $\mathrm{VA}^{30}$. La incomodidad con la idea de la VA se extiende a los clínicos debido a la falta de confianza en la calidad de la literatura, la creencia de que los pacientes estarán más satisfechos con la terapia quirúrgica y también el temor a un potencial desarrollo de metástasis ${ }^{31}$. Una reciente revisión sistemática de casos tratados mediante VA reconoció bajos niveles de progresión tumoral (5,3\%) y metástasis en los ganglios linfáticos $(1,6 \%)$ a 5 años de seguimiento, pero informó que el número de cirugías que se realizaron a los pacientes en VA fue mucho más alto de lo que se esperaba en vista de las bajas cifras ${ }^{52}$.

En Estados Unidos, en vista de la dificultad de evitar el tratamiento excesivo para cáncer de tiroides de bajo riesgo, se han hecho esfuerzos por reducir el número de diagnósticos de tumores de bajo riesgo que incluso ha conllevado a que el grupo de trabajo de servicios preventivos de los Estados Unidos considere que la detección temprana del cáncer de tiroides tiene un efecto negativo neto (Recomendación D) $)^{53}$. En la misma línea, la ATA recomienda no realizar biopsias de nódulos tiroideos de menos de $1 \mathrm{~cm}$ de tamaño, incluso a pesar de que las características del nódulo sean sospechosas de $\mathrm{CPT}^{29}$. Del mismo modo, el sistema de clasificación ecográfica de nódulos tiroideos TIRADS (Thyroid Imaging Reporting and Data System) desaconseja la PAAF para nódulos menores a 1 centímetro no sospechosos ${ }^{54}$. 
Por otro lado, aunque la selección apropiada de los pacientes es invariablemente crítica para realizar la VA, deben tenerse en cuenta otros aspectos al instituir esta modalidad de tratamiento. La experiencia y la comodidad del equipo clínico es primordial y lo ideal sería que incluyera miembros con experiencia en cuidados multidisciplinarios tanto cirujanos como endocrinólogos. La disponibilidad y el uso de la ecografía de cuello de alta calidad para las evaluaciones basales y de seguimiento, y la comprensión de la cinética tumoral son factores invaluables para tomar decisiones terapéuticas. También es importante la planificación apropiada para el seguimiento de los pacientes.

La guía ATA acepta a la VA como una alternativa terapéutica dentro del estándar de cuidado no considerándose como tratamiento experimental. No obstante, la VA es un abordaje novedoso en muchos centros y las experiencias pueden variar entre las diversas instituciones y poblaciones. Por este motivo es importante formalizar la manera en que se vigila a los pacientes con VA a través de la elaboración de protocolos clínicos institucionales que definan los criterios estrictos bajo los cuales los pacientes serán vigilados periódicamente, como también para hacer un seguimiento prospectivo de los datos clínicos e imagenológicos de los pacientes y de reuniones multidisciplinarias recurrentes para evaluar a los pacientes ${ }^{32,52}$.

Una parte importante de los protocolos clínicos institucionales de VA es la discusión que debe tener lugar entre el médico y el paciente. Como implica la terminología "vigilancia activa", ésta trata de un manejo que requiere que el paciente participe activamente. La decisión de llevar a cabo la VA en lugar de una cirugía inmediata debe tomarse después de un proceso de toma de decisiones compartida, una discusión franca y honesta de las opciones y una confianza mutua en la que participen tanto el paciente como el médico. Es imperativo que los pacientes entiendan las pruebas y el razonamiento que hay detrás de la VA, es decir, evitar la cirugía innecesaria y los tratamientos adyuvantes que no es probable que tengan un impacto significativo en la supervivencia y en los resultados específicos de la enfermedad, y evitar la posible morbilidad asociada a esos tratamientos $^{32}$.
Dada la naturaleza de la VA los pacientes deben estar totalmente comprometidos con el proceso y confiar en sus opciones de tratamiento. Es en esta coyuntura que el cirujano juega un papel crítico para ayudar a los pacientes a entender sus opciones. Muchos pacientes pueden llegar a la conclusión de que los riesgos de la cirugía, aunque son bajos, superan sus beneficios, y en particular al tener en cuenta que es posible realizar cirugías igualmente curativas en una fecha posterior si el cáncer de tiroides muestra signos de crecimiento o progresión. Al contrario, otros pacientes pueden concluir que los riesgos de la cirugía son aceptables y preferibles a someterse a un proceso de VA.

\section{Conclusión}

La sólida evidencia acumulada en los estudios realizados hasta la fecha, inicialmente en Japón y más tarde en diversos países con situaciones socioeconómicas y culturales distintas, han permitido conocer la historia natural y el comportamiento biológico de estos tumores. Mediante las extensas experiencias, se ha evidenciado que la gran mayoría de estos tumores no presentan una variación significativa de su tamaño e incluso algunos disminuyen.

La incidencia de eventos adversos es mayor en los pacientes tratados mediante un abordaje quirúrgico temprano que en los pacientes sometidos a VA a pesar de que sean realizadas por cirujanos expertos en patología tiroidea en un centro de alto volumen. Además, se ha reportado en distintas regiones geográficas que los costos médicos del tratamiento quirúrgico resultan muy superiores a los de la VA.

Los criterios de selección inicial de los pacientes para VA deben incluir las características tumorales y de los pacientes, como también la disponibilidad de equipos médicos especializados. Los estudios imagenológicos, las mediciones de biomarcadores fiables y el estudio de la cinética tumoral pueden ser útiles para predecir tempranamente cuáles CPT presentarán mayor riesgo de progresar en el futuro. De esta manera, los pacientes con alto riesgo de progresión podrían ser sometidos a una cirugía inmediata en lugar de VA y los que presenten baja probabilidad de progresión ser tratados mediante VA con una menor fre- 
cuencia de seguimiento ajustada al riesgo. En consecuencia, las investigaciones futuras deben centrarse en el desarrollo de nuevos marcadores tempranos de predicción de la progresión del CPT durante la VA.

A pesar de los resultados alentadores la aceptación de la VA en la práctica clínica sigue siendo limitada. En los países emergentes, los fenómenos socioeconómicos y culturales tales como el menor acceso a equipos especializados de salud, ultrasonido de cuello de alta calidad, y resistencia al cambio por parte de algunos médicos, podrían significar mayor dificultad para el desarrollo y práctica de la VA, mas no su imposibilidad. Los pacientes están fuertemente influenciados por la familia, amigos y sus propios valores. En este sentido, la comunicación eficaz entre el equipo médico y el paciente, conocer las ventajas y desventajas de ambas alternativas terapéuticas y comprender que existe más de un tratamiento correcto, constituye un instrumento esencial para mejorar la aceptación de este abordaje.

\section{Bibliografía}

1. Lim H, Devesa SS, Sosa JA, et al. Trends in thyroid cancer incidence and mortality in the United States, 1974-2013. JAMA. 2017; 317:1338-1348.

2. Ahn HS, Kim HJ, Welch HG. Korea's thyroid cancer "epidemic" - screening and overdiagnosis. N Engl J Med. 2014; 371:1765-1767.

3. Lee JH, Shin SW. Overdiagnosis and screening for thyroid cancer in Korea. Lancet. 2014; 384:1848.

4. Davies L, Welch HG. Increasing incidence of thyroid cancer in the United States, 19732002. JAMA. 2006;295(18):2164-2167. doi:10.1001/ jama.295.18.2164

5. Davies L, Ouellette M, Hunter M, Welch HG. The increasing incidence of small thyroid cancers: where are the cases coming from? Laryngoscope. 2010; 120:2446-2451.

6. Harach HR, Franssila KO, Wasenius VM. Occult papillary carcinoma of the thyroid. A "normal" finding in Finland. A systematic autopsy study. Cancer. 1985; 56(3):531-538.

7. Ito $\mathrm{Y}$, Uruno T, Nakano K, et al. An observation trial without surgical treatment in patients with papillary microcarcinoma of the thyroid. Thyroid 2003; 13(4): 381-7.

8. Shaha AR, Tuttle RM. Editorial: Risk of disease progression during active surveillance of papillary thyroid cancer. Surgery. 2018; 163:53-54.

9. Ito Y, Miyauchi A, Kobayashi K, Miya A. Prognosis and growth activity depend on patient age in clinical and subclinical papillary thyroid carcinoma. Endocr $J$. 2014; 61(3):205-213.

10. Lubitz CC, Kong CY, McMahon PM, et al. Annual financial impact of well differentiated thyroid cancer care in the United States. Cancer. 2014; 120:13451352.

11. Nam-Goong IS, Kim HY, Gong G, et al. Ultrasonography guided fine-needle aspiration of thyroid incidentaloma: correlation with pathological findings. Clin Endocrinol. 2004; 60(1):21-8.

12. Kang HW, No JH, Chung JH, et al. Prevalence, clinical and ultrasonographic characteristics of thyroid incidentalomas. Thyroid. 2004; 14(1):29-33.

13. Haugen BR, Alexander EK, Bible KC, et al. 2015 American Thyroid Association management guidelines for adult patients with thyroid nodules and differentiated thyroid cancer: the American Thyroid Association guidelines task force on thyroid nodules and differentiated thyroid cancer. Thyroid. 2016; 26(1):1-133.

14. Ito $\mathrm{Y}$, Miyauchi A, Kihara M, Higashiyama T, Kobayashi K, Miya A. Patient age is significantly related to the progression of papillary microcarcinoma of the thyroid under observation. Thyroid. 2014; 24(1):27-34.

15. Smulever A, Pitoia F. Active surveillance in papillary thyroid carcinoma: not easily accepted but possible in Latin America. Arch Endocrinol Metab. 2019; 63:462469.

16. Kwon H, Oh HS, Kim M, et al. Active surveillance for patients with papillary thyroid microcarcinoma: a single center's experience in Korea. J Clin Endocrinol Metab. 2017; 102(6):1917-1925.

17. Tuttle RM, Fagin JA, Minkowitz G, et al. Natural history and tumor volume kinetics of papillary thyroid cancers during active surveillance. JAMA Otolaryngol Head Neck Surg. 2017; 143(10):1015-20.

18. Oh HS, Ha J, Kim HI, et al. Active surveillance of low-risk papillary thyroid microcarcinoma: a multicenter cohort study in Korea. Thyroid. 2018; 28:15871594.

19. Oh HS, Kwon H, Song E, et al. Tumor volume doubling time in active surveillance of papillary thyroid carcinoma. Thyroid. 2019; 29(5):642-9.

20. Miyauchi A, Kudo T, Ito Y, et al. Estimation of the lifetime probability of disease progression of papillary microcarcinoma of the thyroid during active surveillance. Surgery. 2018; 163(1):48-52.

21. Miyauchi A, Kudo T, Miya A, et al. Prognostic impact of serum thyroglobulin doubling-time under thyrotropin suppression in patients with papillary thyroid carcinoma who underwent total thyroidectomy. Thyroid. 2011; 21:707-716.

22. Miyauchi A, Kudo T, Ito Y, et al. Natural history of papillary thyroid microcarcinoma: kinetic analyses on 
tumor volume during active surveillance and before presentation. Surgery. 2019; 165:25-30.

23. Lang BH, Wong CK. A cost-effectiveness comparison between early surgery and nonsurgical approach for incidental papillary thyroid microcarcinoma. Eur J Endocrinol. 2015; 173:367-375.

24. Oda H, Miyauchi A, Ito Y, et al. Comparison of the costs of active surveillance and immediate surgery in the management of low-risk papillary microcarcinoma of the thyroid. Endocr J. 2017;64(1):59-64.

25. Venkatesh S, Pasternak JD, Beninato T, et al. Cost-effectiveness of active surveillance versus hemithyroidectomy for micropapillary thyroid cancer. Surgery. 2017; 161:116-126.

26. Aschebrook-Kilfoy B, James B, Nagar S, et al. Risk factors for decreased quality of life in thyroid cancer survivors: initial findings from the North American thyroid cancer survivorship study. Thyroid. 2015; 25:1313-1321.

27. Ito $\mathrm{Y}$, Miyauchi A, Inoue $\mathrm{H}$, et al. An observational trial for papillary thyroid microcarcinoma in Japanese patients. World J Surg. 2010; 34(1):28-35.

28. Jeon MJ, Lee YM, Sung TY, et al. Quality of life in patients with papillary thyroid microcarcinoma managed by active surveillance or lobectomy: a crosssectional study. Thyroid. 2019; 29:956-962.

29. Davies L, Roman BR, Fukushima M, et al. Patient experience of thyroid cancer active surveillance in Japan. JAMA Otolaryngol Head Neck Surg. 2019; 145:363-370.

30. Nickel B, Brito JP, Moynihan R, et al. Patients' experiences of diagnosis and management of papillary thyroid microcarcinoma: a qualitative study. BMC Cancer. 2018; 18:242.

31. Nickel B, Brito JP, Barratt A, et al. Clinicians' views on management and terminology for papillary thyroid microcarcinoma: a qualitative study. Thyroid. 2017; 27: 661-671.

32. Ito Y, Miyauchi A, Kudo T, et al. Trends in the implementation of active surveillance for low-risk papillary thyroid microcarcinomas at Kuma Hospital: gradual increase and heterogeneity in the acceptance of this new management option. Thyroid. 2018; 28:488-495.

33. Sugitani I, Toda K, Yamada K, et al. Three distinctly different kinds of papillary thyroid microcarcinoma should be recognized: our treatment strategies and outcomes. World J Surg. 2010; 34:1222-1231.

34. Brito JP, Moon JH, Zeuren R, et al. Thyroid cancer treatment choice: a pilot study of a tool to facilitate conversations with patients with papillary microcarcinomas considering treatment options. Thyroid. 2018; 28:1325-1331.

35. Oda H, Miyauchi A, Ito $\mathrm{Y}$, et al. Incidences of unfavorable events in the management of low-risk papillary microcarcinoma of the thyroid by active surveillance versus immediate surgery. Thyroid. 2016; 26:150-155.

36. Youngwirth LM, Adam MA, Scheri RP, Roman SA, Sosa JA. Patients treated at low-volume centers have higher rates of incomplete resection and compromised outcomes: analysis of 31,129 patients with papillary thyroid cancer. Ann Surg Oncol. 2016; 23(2):403-9.

37. Griffin A, Brito JP, Bahl M, Hoang JK. Applying criteria of active surveillance to low-risk papillary thyroid cancer over a decade: how many surgeries and complications can be avoided? Thyroid. 2017; 27(4):518-523.

38. Jeon MJ, Kim WG, Choi YM, et al. Features predictive of distant metastasis in papillary thyroid microcarcinomas. Thyroid. 2016; 26(1):161-168.

39. Jeon MJ, Kim WG, Kwon H, et al. Clinical outcomes after delayed thyroid surgery in patients with papillary thyroid microcarcinoma. Eur J Endocrinol. 2017; 177(1):25-31.

40. Kwon H, Jeon MJ, Kim WG, et al. A comparison of lobectomy and total thyroidectomy in patients with papillary thyroid microcarcinoma: a retrospective individual risk factor-matched cohort study. Eur J Endocrinol. 2017; 176(4):371-8.

41. Kwon H, Jeon MJ, Kim WG, et al. Lack of efficacy of radioiodine remnant ablation for papillary thyroid microcarcinoma: verification using inverse probability of treatment weighting. Ann Surg Oncol. 2017; 24(9):2596-602.

42. Park S, Kim WG, Han M, et al. Thyrotropin suppressive therapy for low-risk small thyroid cancer: a propensity score-matched cohort study. Thyroid. 2017; 27(9):1164-70.

43. Kim TY, Kim WB, Song JY, et al. The BRAF mutation is not associated with poor prognostic factors in Korean patients with conventional papillary thyroid microcarcinoma. Horumon To Rinsho. 2005; 63(5):588-93.

44. Yabuta T, Matsuse M, Hirokawa M, Yamashita S, Mitsutake N, Miyauchi A. TERT promoter mutations were not found in papillary thyroid microcarcinomas that showed disease progression on active surveillance. Thyroid. 2017; 27(9):1206-7.

45. Hirokawa M, Kudo T, Ota H, et al. Pathological characteristics of low-risk papillary thyroid microcarcinoma with progression during active surveillance. Endocr J. 2016; 63:805-810.

46. Sugitani I, Fujimoto Y, Yamada K. Association between serum thyrotropin concentration and growth of asymptomatic papillary thyroid microcarcinoma. World J Surg. 2014; 38:673-678.

47. Kim HI, Jang HW, Ahn HS, et al. High serum TSH level is associated with progression of papillary thyroid microcarcinoma during active surveillance. $J$ Clin Endocrinol Metab. 2018;103: 446-451.

48. Ho AS, Daskivich TJ, Sacks WL, Zumsteg ZS. Parallels 


\section{ARTíCULO DE REVISIÓN}

between low-risk prostate cancer and thyroid cancer: a review. JAMA Oncol. 2019; 5:556-564.

49. Lowenstein LM, Basourakos SP, Williams MD, et al. Active surveillance for prostate and thyroid cancers: evolution in clinical paradigms and lessons learned. Nat Rev Clin Oncol. 2019; 16(3):168-84.

50. Haugen BR, Alexander EK, Bible KC, et al. 2015 American Thyroid Association management guidelines for adult patients with thyroid nodules and differentiated thyroid cancer: the American Thyroid Association guidelines task force on thyroid nodules and differentiated thyroid cancer. Thyroid. 2016; 26(1):1-133.

51. Ho AS, Chen I, Melany M, Sacks WL. Evolving management considerations in active surveillance for micropapillary thyroid carcinoma. Curr Opin Endocrinol Diabetes Obes. 2018; 25:353-359.

52. Cho SJ, Suh CH, Baek JH, et al. Active surveillance for small papillary thyroid cancer: a systematic review and meta-analysis. Thyroid. 2019; doi: 10.1089/ thy.2019.0159.

53. Bibbins-Domingo K, Grossman DC, Curry SJ, et al. Screening for thyroid cancer: US preventive services task force recommendation statement. JAMA. 2017; 317:1882-1887.

54. Grant EG, Tessler FN, Hoang JK, et al. Thyroid ultrasound reporting lexicon: white paper of the ACR thyroid imaging, reporting and data system (TIRADS) committee. J Am Coll Radiol. 2015; 12:1272-1279. 